\title{
Self-perception of teaching fellows and learning assistants in introductory physics classes
}

\author{
Alexander P. Becker, Bennett Goldberg, and Manher Jariwala \\ Department of Physics, Boston University, 590 Commonwealth Avenue, Boston, MA 02215
}

\begin{abstract}
We study how graduate student teaching fellows (TFs) and undergraduate learning assistants (LAs) view their roles and responsibilities as educators in undergraduate classrooms. We present results from a survey of 35 physics TFs and LAs across a range of physics classes, measuring their expectations of their teaching mission with regard to such factors as classroom authority, student interaction time, responsibility for student learning, and helpfulness to students. We further analyze their answers based on the classroom format they have taught in. We find that the perceptions TFs and LAs express in the survey regarding their roles in the classroom are similar; however, we find differences when looking at the questions surrounding teacher-student interactions.
\end{abstract}

\section{INTRODUCTION}

During graduate school, physics PhD students usually serve as teaching fellows (TFs, or equivalently TAs) in their department. They help teach undergraduate physics classes, hold discussion sections, labs, and/or office hours and grade student work. The introduction of learning assistants (LAs) has transformed the classroom experience in which teaching fellows teach [1]. These undergraduate students serve as near-peer instructors and are physics majors or have previously taken the class to which they are assigned themselves.

At Boston University, LAs in the physics department either support the professor teaching lecture and the TF in leading discussion, or alternatively they are engaged together with the professor and TFs in the studio or "SCALE-UP" setting [2], which fuses lecture, lab and discussion. Research has been devoted to investigating the outcomes for undergraduates who become learning assistants and the impact of teaching on graduate students [3, 4]. As educational reforms in physics instruction become more widespread, more attention has been paid to the beliefs that teaching fellows have toward teaching and learning, and how that affects their practices and the implementation of these reforms [5-7]. With this paper, we want to understand the perception TFs as well as LAs at Boston University in different class formats have of themselves in the classroom, adding a comparative component to existing work. Using a pre- and post-semester survey, we assess how teaching fellows and learning assistants see their own roles as well as that of other instructors.

BU physics is currently transforming its biggest introductory classes for premeds and engineers from the classical lecture-lab-discussion format to studio. While research suggests favorable learning outcomes for students [8], we want to study here the impact this setting has on the TFs and LAs.

\section{METHODS}

The teaching mission of BU's physics department includes introductory physics courses for health science, life science, physical science and engineering majors. 32-33 graduate students serve as teaching fellows, and another 20-21 undergraduate students (a mix of physics and other majors) as learning assistants. Classes are taught in both the classical format of lecture supplemented by labs and discussion groups, which we term LLD, and the studio format. In the LLD format, professors teach the lecture which is attended by the undergraduate learning assistants in the course. The TFs and LAs teach discussion sections together, and labs are led by TFs alone. In the studio classroom, professors, TFs and LAs jointly teach the class, at the same time, together in one room.

All TFs and LAs were given an online survey at the beginning of the Spring 2016 semester, asking about their expectations specifically for the upcoming teaching assignment. At the end of the semester, as classes had concluded, they were given the same questions again, inquiring about their experiences that semester. The questions are shown in Table I.

TABLE I. Survey questions.

\begin{tabular}{l|l}
\hline \hline No. & Question \\
\hline Q1 & $\begin{array}{l}\text { How is the time students spend in interaction with profes- } \\
\text { sors, TFs, and LAs distributed? } \\
\text { Q2a }\end{array}$ \\
Q2b & $\begin{array}{l}\text { How much responsibility do professors, TFs, and LAs have } \\
\text { Tor student learning? } \\
\text { To what extent can professors, TFs, and LAs positively in- } \\
\text { fluence how well students learn? }\end{array}$ \\
Q3a & $\begin{array}{l}\text { How much authority do professors, TFs, and LAs have over } \\
\text { the course and its design? }\end{array}$ \\
Q3b & $\begin{array}{l}\text { How much authority do professors, TFs, and LAs have in } \\
\text { the classroom? }\end{array}$ \\
Q4a & $\begin{array}{l}\text { How much of the workload of the course and its design do } \\
\text { professors, TFs, and LAs carry? }\end{array}$ \\
Q4b & $\begin{array}{l}\text { How much of the workload do professors, TFs, and LAs } \\
\text { carry in the classroom? }\end{array}$ \\
Q5a & $\begin{array}{l}\text { In your view, how much do students think of professors, } \\
\text { TFs, and LAs as a helpful resource? } \\
\text { In your view, how much do students think of professors, } \\
\text { TFs, and LAs as a relatable resource? }\end{array}$ \\
Q5b
\end{tabular}

Each of the possible variables, that is, professors, teaching fellows and learning assistants, was to be assigned a numerical value with a slider. The slider took values from 0 to 100 , set to 0 initially. The sum of the three sliders had to equal 100 , and the survey software forced a correction if this constraint was not met. Figure 1 shows an image of the survey; the sliders are non-zero for illustration purposes. 
Having to give a total of 100 points, the survey respondents effectively assigned percentages to professors, TFs and LAs for each question. The participants were instructed that, e.g., if asked about the responsibility for student learning, an assigned score of 0 would mean they view that group to have no responsibility, and an assigned score of 100 would mean they view that group carries the complete responsibility.

Based on your experience from Spring 2016, how much responsibility did professors, TFs, and LAs have for student learning?

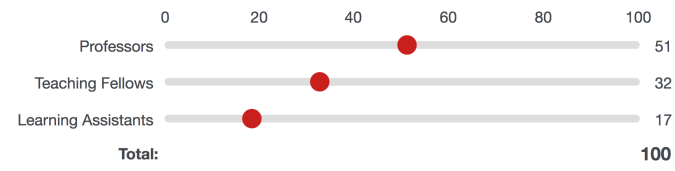

FIG. 1. Example from the online survey, illustrating the sliders.

We recorded 40 individual responses. A total of $20 \mathrm{TFs}$ and 15 LAs took both the pre- and post-semester survey. We analyze the matched set of these 35 respondents in order to compare between expectations before and the experiences during their teaching assignment. This small sample leads to larger error bars associated with the results; the standard deviation is generally in the range of 5 to 15 percentage points.

We also polled the participants about their teaching assignment and previous experience. Additionally we asked them about their attitude towards teaching before their teaching assignment and to reflect on their experience during the semester. Answers to these questions were to be given on a scale from 1 to 5 , as is detailed below. In the pre-survey, TFs and LAs had to self-assess their enthusiasm for teaching from "not at all enthusiastic" to "very enthusiastic." In the post-survey, we asked the participants if and how the past semester had changed their enthusiasm, from "much less enthusiastic now" to "much more enthusiastic now." Furthermore the participants were asked to rate their recent teaching experience, from "much worse than expected" to "much better than expected." Finally, TFs and LAs had to self-assess their development as a teacher, agreeing or disagreeing with the statement "I have become a better teacher this semester."

\section{RESULTS}

We observe that the average values TFs and LAs assign in the pre-semester survey are comparable to those in the post-semester survey. Generally, we found two different percentage distributions characterized by participant responses to the survey items. In one case, respondents assign roughly 50-70 percent to professors, significantly less than 20 percent to LAs, and the rest to TFs. These questions survey the attitude towards the responsibility and workload within the framework of the entire course. In the other case, respondents assign roughly 40-55 percent to professors, $20-35$ percent to TFs and up to 25 percent to the LAs, with one notable exception which we will discuss further below. These questions deal with the instructor-student interaction in the classroom.
TABLE II. Comparing pre- and post-means for selected questions.

\begin{tabular}{cccc}
\hline \hline Question & Group & Pre-Survey & Post-Survey \\
\hline \multirow{2}{*}{ Q2a } & P & 54 & 51 \\
& TF & 31 & 32 \\
& LA & 15 & 17 \\
\hline \multirow{2}{*}{ Q2b } & P & 48 & 46 \\
& TF & 31 & 33 \\
& LA & 21 & 21 \\
\hline \multirow{2}{*}{ Q3b } & P & 56 & 60 \\
& TF & 31 & 28 \\
& LA & 13 & 12 \\
\hline \multirow{2}{*}{ Q5a } & P & 44 & 42 \\
& TF & 36 & 36 \\
\hline \multirow{2}{*}{ Q5b } & LA & 20 & 22 \\
& P & 31 & 35 \\
& TF & 34 & 35 \\
\hline \hline
\end{tabular}

In our analysis, we focus on questions Q2a and Q2b, Q3b, as well as Q5a and Q5b. They are referring to the responsibility and influence over student learning, the authority in the classroom, and the perceived helpfulness and relatability, respectively. We can categorize them as questions about the teacher-student interaction. In Table II we present the mean pre- and post-semester results for these questions, and again we want to point out that the values stay roughly the same.

It is noteworthy that question Q5b records the highest value for learning assistants across all questions. After the semester, this shifts slightly, but the vote is still split fairly evenly. Despite falling behind professors and TFs, the postsurvey value for LAs is still their highest of all questions.

In the following, we disaggegrate the responses. We analyze the five questions listed above with regard to whether respondents are TFs or LAs and the format they teach.

\section{A. Grouping teaching fellows and learning assistants}

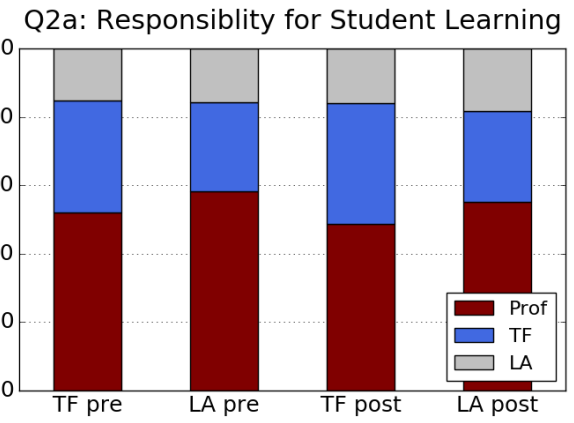

FIG. 2. Perceived responsibility for student learning, differentiating between TF and LA responses.

In order to gain insight into the self-perception of teaching fellows and learning assistants and their view of other instructors, we break down the results by TF and LA status. TFs and LAs perceive the distribution of responsibility for 
student learning differently. Figure 2 shows that teaching fellows assign themselves more responsibility than LAs do. The values for LAs, however, are the same, regardless of respondent group or time of the survey, pre or post. This suggests that teaching fellows and learning assistants have different impressions of the roles of TFs as compared to professors, but agree on the roles of LAs. Interestingly the results for authority in the classroom (Q3b) and workload in the classroom (Q4b) look almost identical (not shown here), and the same conclusions seem to apply. We may extend this finding to Q5a, asking how much students would think of the instructors as a helpful resource. As shown in Fig. 3, the values for teaching fellows and learning assistants are slightly higher than in the previously discussed questions, at the expense of professors. It is striking that TFs think they are viewed as significantly more helpful than LAs. The opposite is not true; at most LAs believe the difference in helpfulness to be less. Table II indicates that LAs achieve a slightly higher value in the post-survey for this question. Disaggregating the data shows that this stems from the LAs' answers only, as TFs notice no changes for LAs.

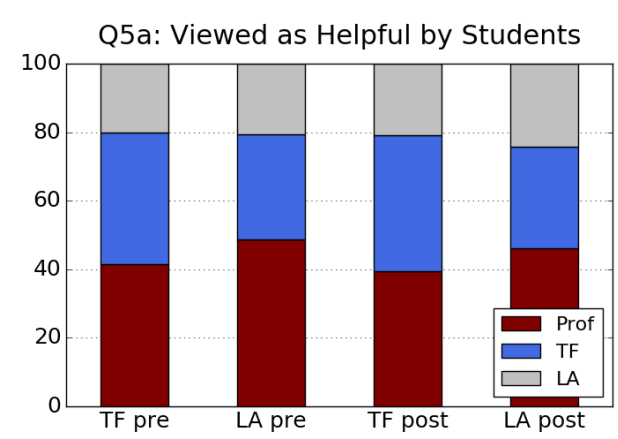

FIG. 3. Estimate by TFs and LAs for how helpful students perceive each kind of instructor to be.

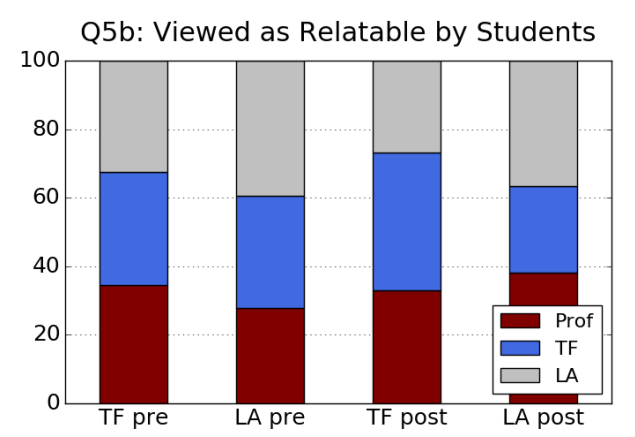

FIG. 4. Estimate by TFs and LAs for how relatable students perceive each kind of instructor to be.

The question for which we recorded the highest scores for learning assistants is Q5b, asking about the relatability of the instructors. Before the semester, teaching fellows expect that students view LAs as very relatable (P 34, TF 33, LA 33). Learning assistants hold this opinion even more strongly, as Figure 4 shows. In fact, LAs expect to be considered most relatable and professors the least relatable (P 26, TF 35, LA 39).
The results of the post-survey exhibit a discrepancy between expectation and experience. Teaching fellows think that they themselves have been viewed as the most relatable group by their students and learning assistants as the least relatable group (P 33, TF 40, LA 27). Despite assigning themselves a lower value in the post-survey, LAs still rank themselves as more relatable than TFs (P 38, TF 27, LA 35).

\section{B. Grouping LLD and studio}

Of the 35 respondents, 25 taught in the classical LLD format and 10 taught in a studio classroom, of which five were teaching fellows and five were learning assistants. In our analysis, we focus on questions Q2b and Q5b, dealing with the influence over student learning and the perceived relatability, as these have shown to be distinguishing aspects in the previous section and we believe them to be critical to the self-perception of graduate and undergraduate instructors. In Figs. 5 and 6 we show the results, disaggregated by class format. It is unsurprising that professors receive higher percentages in the studio format than in the LLD format for both of these questions because TFs and LAs teach together with them in the same room. This holds equally true for pre- and post-survey. These higher values come to a large extent at the expense of the percentages assigned to LAs.

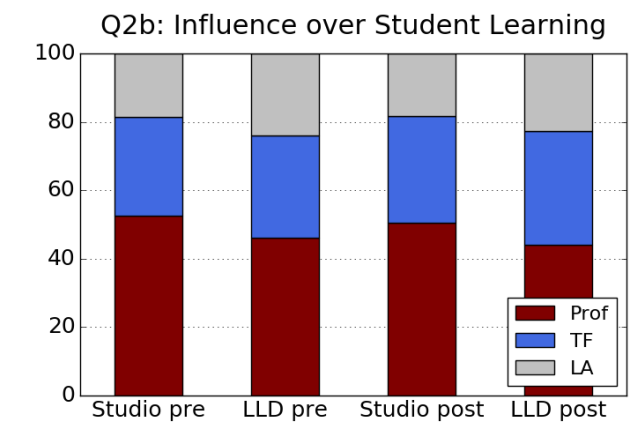

FIG. 5. Influence of instructors over student learning, broken down according to the format in which the respondents teach.

Let us first consider question Q2b in more detail, on the influence over student learning. Pre- and post-semester survey yield very similar results, therefore we will consider the pre-semester numbers now. In the studio format, professors are assigned 53 percent, whereas in LLD the number is only 46 percent. Teaching fellows are assigned 31 percent in the studio, but 34 percent in LLD; similarly TAs get 18 and 22 percent, respectively.

This contrast is more striking when considering the relatability. Instructors teaching in the format with lecture, lab and discussion believe that LAs are viewed as most relatable by the students, while professors are thought to be viewed as least relatable. We find an even split for the studio format in the pre-survey and the opposite result in the post-survey, as Fig. 6 shows. Recalling the overall decrease in relatability for LAs in the post-survey from Table II, we recognize that this change appears to be independent of class format. 
Instructors in LLD and in studio reported a slight increase in their enthusiasm for teaching (3.4 out of 5). On average TFs and LAs in the studio format concluded that it went slightly better than expected (3.4 out of 5), whereas TFs and LAs in LLD found their expectations met (3.1). Despite this, TFs and LAs in LLD assess themselves to have improved notably (4.0 out of 5), whereas their counterparts in studio are less inclined to consider themselves better teachers (3.4).

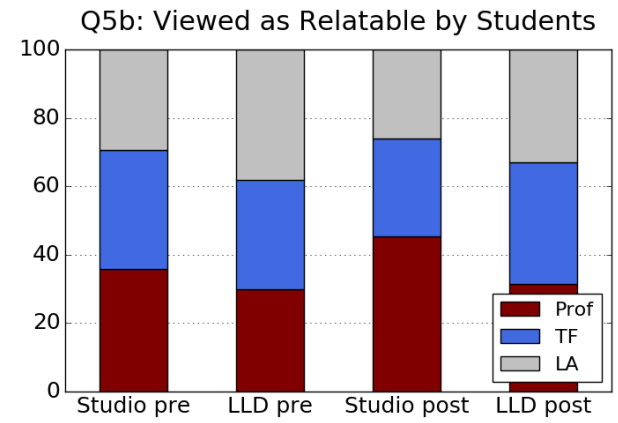

FIG. 6. Disaggregation of perceived helpfulness by class format.

\section{DISCUSSION}

Teaching fellows and learning assistants believe the roles of professors, TFs and LAs in the classroom to be generally similar. This holds true for pre- and post-survey. But when focusing on student-teacher interactions, notable differences appear. TFs assign themselves higher responsibility and influence over student-learning and see themselves on par with professors considering the interaction time in the classroom and as how helpful students view them. They assign equal values to the LAs as LAs assign to themselves. However, LAs perceive the role of the professor to be much larger than that of TFs. While LAs, serving as near-peer instructors, believe to be seen as the most relatable by students, they think that both professors and TFs are perceived as almost equally relatable. On top of that, post-teaching LAs believe they were viewed as less relatable than they had expected.

The analysis of the self-perception of instructors in different classroom settings may point to the following hypothesis: while improving student learning gains, studio classrooms are less helpful to learning assistants in their teaching development than the classical format of lecture, lab and discussion. LAs are assigned lower values across the board in questions concerning student-teacher interaction; at the same time the numbers for TFs remain almost the same. In a selfassessment after the semester, instructors in LLD found themselves to be better teachers more so than instructors in studio. This is despite the fact that instructors in studio classrooms perceived their assignment, on average, to have gone slightly better than expected, unlike their counterparts in LLD.

We propose two possible explanations. For one thing, TFs and LAs in LLD teach the same material multiple times a week in different sections. This allows them to practice more, evaluate their teaching and recognize and apply approaches that help students best. In studio, the material changes from session to session. For another thing, TFs and LAs in LLD are more autonomous and teach without the presence of a professor. This is very different in studio where the graduate and undergraduate instructors see the professors in student interactions comparable to their own. Possibly the professor is perceived as an overbearing presence, or TFs and LAs recognize the teaching skills of their professor better than in LLD.

Since our sample has been small, the differences in responses we have identified are not generally statistically significant. We are addressing this issue by repeating this survey in the Fall semester. Furthermore we plan to conduct interviews at the end of this semester.

\section{CONCLUSION}

We have utilized a novel survey tool to explore the selfperceptions of TFs and LAs in the classroom, demonstrating both anticipated as well as surprising results regarding their interactions with students. We suggest that further research on active-learning classrooms should not only be dedicated to student outcomes, but also to the effects on the development of TFs and LAs as teachers, since many LA programs are installed as a means to produce more physics teachers.

\section{ACKNOWLEDGMENTS}

This work was funded in part by NSF-IUSE Grant No. DUE-1525354 and CIRTL-GLHE Grant No. 581K055. This paper is Contribution No. LAA-041 of the International Learning Assistant Alliance.
[1] V. Otero, S. Pollock, and N. Finkelstein, American Journal of Physics 78 (2010).

[2] R. Beichner, Invited paper for the National Academy of Sciences (2008).

[3] E. Close, J. Conn, and H. Close, Physical Review Physics Education Research 12 (2016).

[4] I. Quitadamo, C. J. Brahler, and G. Crouch, Science Educator 18 (2009).
[5] R. M. Goertzen, R. E. Scherr, and A. Elby, Phys. Rev. ST Phys. Educ. Res. 5, 020109 (2009).

[6] R. M. Goertzen, R. E. Scherr, and A. Elby, Phys. Rev. ST Phys. Educ. Res. 6, 020125 (2010).

[7] B. T. Spike and N. D. Finkelstein, Phys. Rev. Phys. Educ. Res. 12, 010114 (2016).

[8] C. Hoellwarth, M. J. Moelter, and R. D. Knight, American Journal of Physics 73, 459 (2005). 